\title{
Localization of spin waves in disordered quantum rotors
}

\author{
Alexei Andreanov ${ }^{1}$ and Andrei A. Fedorenko ${ }^{2}$ \\ ${ }^{1}$ Max-Planck-Institut für Physik komplexer Systeme, Nöthnitzer Straße 38, D-01187 Dresden, Germany \\ ${ }^{2}$ CNRS UMR5672 - Laboratoire de Physique de l'Ecole Normale Supérieure de Lyon, 46, Allée d'Italie, 69007 Lyon, France
}

(Dated: October 3, 2018)

\begin{abstract}
We study the dynamics of excitations in a system of $O(N)$ quantum rotors in the presence of random fields and random anisotropies. Below the lower critical dimension $d_{\mathrm{lc}}=4$ the system exhibits a quasi-long-range order with a power-law decay of correlations. At zero temperature the spin waves are localized at the length scale $L_{\mathrm{loc}}$ beyond which the quantum tunneling is exponentially suppressed $c \sim e^{-\left(L / L_{\mathrm{loc}}\right)^{2(\theta+1)}}$. At finite temperature $T$ the spin waves propagate by thermal activation over energy barriers that scale as $L^{\theta}$. Above $d_{\mathrm{lc}}$ the system undergoes an order-disorder phase transition with activated dynamics such that the relaxation time grows with the correlation length $\xi$ as $\tau \sim e^{C \xi^{\theta} / T}$ at finite temperature and as $\tau \sim e^{C^{\prime} \xi^{2(\theta+1)} / \hbar^{2}}$ in the vicinity of the quantum critical point.

PACS numbers: 71.55.Jv, 75.10.Nr, 05.30.Rt
\end{abstract}

\section{INTRODUCTION}

Localization of excitations in disordered quantum systems has been attracting considerable interest during the last several decades. While single particle localization is rather well understood within the standard theory of Anderson localization, ${ }^{1}$ localization of interacting particles is a much more complicated problem where many questions remain open. ${ }^{2-4} \mathrm{Re}-$ cently, excited many-body localized eigenstates were studied in the random field Heisenberg spin- $\frac{1}{2}$ chain using exact diagonalization $^{5}$ and in the random anisotropy $X X Z$ spin- $\frac{1}{2}$ chain by applying a dynamical real space renormalization group. ${ }^{6}$ It was found that the many-body localized states in closed quantum systems with quenched randomness share many properties with quantum glasses, e.g., they fail to thermally equilibrate and break ergodicity. It was argued that such systems can be described by an infinite-randomness fixed point (FP) with an infinite dynamic critical exponent. ${ }^{4-6}$ Unlike fermions, bosons can condense into a superfluid state with long-range order so that interactions are intrinsically unavoidable. The presence of disorder can suppress the phase coherence of the bosons and localize them collectively in a compressible Bose glass with a gapless energy spectrum ${ }^{7-10}$ or in an incompressible Mott glass. ${ }^{11-13}$ The zero-temperature superfluid-insulator transition in two-dimensional disordered hard-core bosons has been recently studied using a spin-wave approach. ${ }^{14}$ A mobility edge in the spin-wave excitation spectrum has been found at a finite frequency that vanishes in the Bose glass phase. The connection between the Bose (Mott) glass and disordered elastic systems has been known for long time. ${ }^{8-10}$ Recently, a mapping of the leading order perturbation theory for boson Green's functions to a directed polymer in random media has been proposed for studying the insulating phase of charged hard-core bosons. ${ }^{15}$

In this paper we investigate the dynamics of a $d$ dimensional system of $O(N)$ quantum rotors in the presence of random fields and random anisotropies. This model shares many properties with the aforementioned systems but allows for an analytical study using the functional renormalization group (FRG) that was originally developed for disor- dered elastic systems such as the directed polymer in random media. ${ }^{16-19}$ The FRG reveals that the behavior of the disordered quantum rotors is controlled by a quasiclassical zerotemperature FP. In the real space renormalization group treatment of spin chains one fixes the temperature and the Planck constant so that the renormalized disorder strength grows approaching an infinite-randomness FP. In our FRG scheme we fix the disorder strength near the FP but allow the temperature and the effective Planck constant to flow to zero. Both parameters turn out to be dangerously irrelevant like the temperature in the random field Ising model. ${ }^{20}$ This drastically changes the dynamic scaling picture that one could expect from a naive $R G$ treatment. ${ }^{21,22}$ The appearance of nonanalyticity in the FRG flow prevents the system from equilibration by inducing activated dynamics with diverging barriers at finite temperature and localization at zero temperature. This mechanism is to some extent similar to the one behind the classical and quantum creep of disordered elastic systems at small driving forces. ${ }^{23,24}$

The paper is organized as follows: We introduce the model in Sec. II and apply the FRG in Sec. III. In Sec. IV we discuss the localization properties of excitations in the quasi-longrange order (QLRO) phase below the lower critical dimension. Section $\mathrm{V}$ is devoted to the activated dynamics at the order-disorder transition above the lower critical dimension. The Appendices present the technical details of the derivation of the FRG flow equations.

\section{MODEL}

The Hamiltonian of interacting quantum rotors on a $d$ dimensional hyper-cubic lattice with lattice constant $b$ can be written as

$$
\mathcal{H}_{0}=\frac{1}{2 I} \sum_{i} \hat{\mathbf{L}}_{i}^{2}-\sum_{\langle i, j\rangle} J_{i j} \hat{\mathbf{n}}_{i} \hat{\mathbf{n}}_{j}, \quad \hat{\mathbf{n}}_{i}^{2}=1,
$$

where the operator $\hat{\mathbf{n}}_{i}$ is a $N$-dimensional unit-length vector representing the orientation of the rotor on site $i . \hat{\mathbf{L}}_{i}$ is the angular momentum operator whose $N(N-1) / 2$ components are 
defined as $\hat{L}_{i \mu \nu}=\hat{n}_{i \mu} \hat{p}_{i \nu}-\hat{n}_{i \nu} \hat{p}_{i \mu}$. The momentum operator of each rotor with the moment of inertia $I$ satisfies the commutation relations $\left[\hat{n}_{i \mu}, \hat{p}_{j \nu}\right]=i \hbar \delta_{i j} \delta_{\mu \nu}$. The first term in (1) is the kinetic energy of the rotor with the moment of inertia $I$. In the case of randomly distributed exchange interactions $J_{i j}$ the system forms a strong quantum glass which has been studied mainly in the limit of infinite range interactions using $1 / N$-expansion. ${ }^{25}$ The limit of $N=1$ is expected to be in the same universality class as the Ising model in a transverse field whose glass phase is critical everywhere and exhibits gapless collective excitations in the long-range interaction limit. ${ }^{26}$ Here we assume that all $J_{i j}=J$ and restrict the sum $\langle i, j\rangle$ to nearest neighbors. Instead of the random exchange interactions we introduce random fields and random anisotropies as $\mathcal{H}=\mathcal{H}_{0}+\mathcal{H}_{\mathrm{RF}}+\mathcal{H}_{\mathrm{RA}}$, where $\mathcal{H}_{\mathrm{RF}}=-\sum_{i} \mathbf{h}_{i} \cdot \hat{\mathbf{n}}_{i}$ and $\mathcal{H}_{\mathrm{RA}}=-\sum_{i}\left(\mathbf{d}_{i} \cdot \hat{\mathbf{n}}_{i}\right)^{2}$ with randomly oriented vectors $\mathbf{h}_{i}$ and $\mathbf{d}_{i}$. In the continuum limit this model can be rewritten as an $O(N)$ quantum-mechanical nonlinear $\sigma$-model (QNL $\sigma \mathrm{M})$ with the partition function $\mathcal{Z}=\int \mathcal{D} \mathbf{n} \delta(|\mathbf{n}|-1) e^{-\mathcal{S}[\mathbf{n}] / \hbar}$ and the imaginary time action

$$
\begin{aligned}
\mathcal{S}[\mathbf{n}] & =\frac{\rho_{0}}{2} \int_{\tau, x}\left[\frac{1}{c_{0}^{2}}\left(\partial_{\tau} \mathbf{n}(\tau, x)\right)^{2}+(\nabla \mathbf{n}(\tau, x))^{2}\right] \\
& -\int_{\tau, x} \sum_{\mu=1}^{\infty} \sum_{i_{1} \cdots i_{\mu}} h_{i_{1} \cdots i_{\mu}}^{(\mu)}(x) n_{i_{1}}(\tau, x) \cdots n_{i_{\mu}}(\tau, x),
\end{aligned}
$$

where we have introduced the shorthand notations $\int_{\tau}:=$ $\int_{0}^{\hbar / T} d \tau$ and $\int_{x}:=\int d^{d} x$. Here $\rho_{0}=b^{2-d} J$ is the bare stiffness constant, $c_{0}=b \sqrt{J / I}$ the bare spin-wave velocity and $T$ the temperature. The UV cutoff $\Lambda_{0}=2 \pi / b$ is imposed in (2). The $O(N)$ QNL $\sigma \mathrm{M}$ arises as an effective theory for the low energy degrees of freedom in several correlated quantum systems. For instance, the $O(2)$ model describes Cooper pairs of electrons in a superconducting Josephson junctions array and ultra-cold atoms in an optical lattice. ${ }^{27}$ The $O(3)$ model describes a quantum spin- $S$ antiferromagnet in the large- $S$ limit. $^{28}$ The $O(5)$ QNL $\sigma \mathrm{M}$ was suggested for the unified lowenergy theory of the antiferromagnetic and superconducting phases in the high- $T_{c}$ superconductors. ${ }^{29}$ The renormalization of the original model (1) with random fields $h^{(1)}$ and/or anisotropies $h^{(2)}$ generates the higher rank anisotropies $h^{(\mu)}$, which we incorporated in the second line of (2) from the beginning. ${ }^{30,31}$ The RG flow preserves the symmetry with respect to inversion $\mathbf{n} \rightarrow-\mathbf{n}$, so we will use the notation of random anisotropy (RA) for the systems respecting this symmetry and random field (RF) for the rest. The bare $\mu$ th rank anisotropies can be taken to be Gaussian distributed with zero mean and cumulants

$$
\overline{h_{i_{1} \cdots i_{\mu}}^{(\mu)}(x) h_{j_{1} \cdots j_{\nu}}^{(\nu)}\left(x^{\prime}\right)}=\delta^{\mu \nu} \delta_{i_{1} j_{1}} \cdots \delta_{i_{\mu} j_{\nu}} r^{(\mu)} \delta\left(x-x^{\prime}\right) .
$$

We use the replica trick to average over disorder. Introducing $n$ replicas of the original system we obtain the replicated action

$$
\begin{aligned}
\mathcal{S}_{n}[\{\mathbf{n}\}]= & \frac{\rho_{0}}{2} \sum_{a=1}^{n} \int_{\tau, x}\left[\frac{1}{c_{0}^{2}}\left(\partial_{\tau} \mathbf{n}_{a}(\tau, x)\right)^{2}+\left(\nabla \mathbf{n}_{a}(\tau, x)\right)^{2}\right] \\
& -\frac{1}{2 \hbar} \sum_{a, b=1}^{n} \int_{\tau, \tau^{\prime}, x} R\left(\mathbf{n}_{a}(\tau, x) \cdot \mathbf{n}_{b}\left(\tau^{\prime}, x\right)\right),
\end{aligned}
$$

where we have introduced $R(z)=\sum_{\mu} r^{(\mu)} z^{\mu}$, which is defined for $-1 \leq z \leq 1$. This function is even for the RA model and has no symmetry for the RF model. The properties of the original disordered system (2) can be extracted in the limit $n \rightarrow 0$. The Imry-Ma arguments suggest that true long-range order is absent in our model for $d<4$, i.e. $d_{\mathrm{lc}}=4$ is the lower critical dimension. However, a quantum QLRO can survive at low enough temperature, similarly to the QLRO in the classical Heisenberg model. ${ }^{32}$ In the QLRO phase the local order slowly changes in space, leading to a power-law decay of correlations that justifies the description of the dynamics in terms of spin-wave excitations.

\section{FUNCTIONAL RENORMALIZATION GROUP}

To get access to the low- $T$ phase we renormalize the action (4) using a momentum-shell method in which iterative integrations over fast modes with wavevectors between the bare cutoff $\Lambda_{0}$ and the running cutoff $\Lambda_{\ell}=\Lambda_{0} e^{-\ell}$ generate the RG flow equations. Dimensional analysis of the action (4) shows that all the derivatives of $R(z)$ at $z=0$ are relevant operators. Thus, one needs to follow the renormalization of the entire function $R(z)$. It is convenient to express the flow equations in terms of the reduced running quantities:

$$
\begin{aligned}
\tilde{R}_{\ell}(\phi) & =K_{d} R_{\ell}(z) \rho_{\ell}^{-2} \Lambda_{\ell}^{d-4}, \\
\tilde{\hbar}_{\ell} & =K_{d} \hbar \rho_{\ell}^{-1} \Lambda_{\ell}^{d-1} \\
\tilde{T}_{\ell} & =K_{d} T_{\ell} \rho_{\ell}^{-1} \Lambda_{\ell}^{d-2}
\end{aligned}
$$

where $z=\cos \phi$ and $K_{d}$ is the surface of the unit sphere in $d$ - dimensions divided by $(2 \pi)^{d}$. The function $\tilde{R}_{\ell}(\phi)$ is $\pi$ periodic for the RA and $2 \pi$-periodic for the RF model. We expand the action around a locally ordered state and neglect the possible presence of topological defects that can modify the behavior of the system. ${ }^{33}$ We split the local order parameter $\mathbf{n}_{a}=\left(\sigma_{a}, \boldsymbol{\pi}_{a}\right)$ into the component $\sigma_{a}=\sqrt{1-\boldsymbol{\pi}_{a}^{2}}$ aligned along the locally preferred direction and the $(N-1)$ component vector $\boldsymbol{\pi}_{a}$ perpendicular to it. We decompose the latter into slowly and rapidly varying parts $\boldsymbol{\pi}_{a}^{<}$and $\boldsymbol{\pi}_{a}^{>}$with the momentum modes $0<q<\Lambda_{\ell}$ and $\Lambda_{\ell}<q<\Lambda_{0}$, respectively. Integrating out the fast fields $\pi_{a}^{>}$and allowing for the rescaling of the slow fields $\boldsymbol{\pi}_{a R}(x)=\zeta \boldsymbol{\pi}_{a}^{<}(x)$ with

$$
\zeta=1+\frac{1}{2}(N-1) \tilde{R}_{\ell}^{\prime \prime}(0) \ell+O\left(\tilde{R}_{\ell}^{2}\right),
$$

we obtain the one-loop flow equations for the effective temperature $\tilde{T}_{\ell}$ and the Planck constant $\tilde{\hbar}_{\ell}$ (see Appendix A for more details)

$$
\partial_{\ell} \ln \tilde{T}_{\ell}=1+\partial_{\ell} \ln \tilde{\hbar}_{\ell}=2-d-(N-2) \tilde{R}_{\ell}^{\prime \prime}(0),
$$




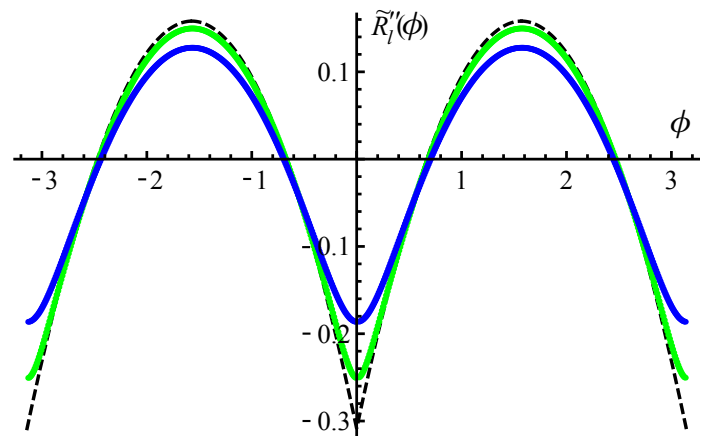

FIG. 1. (Color online) The $\pi$ - periodic FP solution $\tilde{R}^{\prime \prime}(\phi)$ describing the QLRO phase in the 3D $O(3) \mathrm{RA}$ model. The dashed line is the zero- $\Gamma$ FP, the solid green and blue lines with a rounded cusp are the finite $\Gamma$ FPs for $\Gamma=0.05$ and $\Gamma=0.1$.

and for the disorder correlator

$$
\begin{aligned}
& \partial_{\ell} \tilde{R}_{\ell}(\phi)=\varepsilon \tilde{R}_{\ell}(\phi)+\tilde{R}_{\ell}^{\prime \prime}(\phi)\left[\Gamma_{\ell}-\tilde{R}_{\ell}^{\prime \prime}(0)\right]+\frac{1}{2}\left[\tilde{R}_{\ell}^{\prime \prime}(\phi)\right]^{2} \\
& +(N-2)\left(\frac{\tilde{R}_{\ell}^{\prime}(\phi)^{2}}{2 \sin ^{2} \phi}+\left[\frac{\tilde{R}_{\ell}^{\prime}(\phi)}{\tan \phi}+2 \tilde{R}_{\ell}(\phi)\right]\left[\Gamma_{\ell}-\tilde{R}_{\ell}^{\prime \prime}(0)\right]\right) .
\end{aligned}
$$

Here we introduced $\varepsilon=4-d$ and the boundary layer width

$$
\Gamma_{\ell}=\frac{1}{2} c_{\ell} \tilde{\hbar}_{\ell} \operatorname{coth}\left[\frac{c_{\ell} \tilde{\hbar}_{\ell}}{2 \tilde{T}_{\ell}}\right]=\left\{\begin{array}{cl}
\tilde{T}_{\ell} & \text { if } \tilde{\hbar}_{\ell} \rightarrow 0, \\
\frac{1}{2} c_{\ell} \tilde{\hbar}_{\ell} & \text { if } \tilde{T}_{\ell} \rightarrow 0,
\end{array}\right.
$$

that describes the joint effect of thermal and quantum fluctuations on the disorder correlator flow. Disorder breaks the Lorentz invariance of the clean system and renormalizes the spin-wave velocity

$$
\partial_{\ell} \ln c_{\ell}=-\frac{1}{6}\left[(N+1) \tilde{R}_{\ell}^{(4)}(0)+(N-2) \tilde{R}_{\ell}^{\prime \prime}(0)\right]
$$

similarly to the stiffness constant in disordered elastic systems with broken statistical tilt symmetry., ${ }^{9} 10$

Assuming that the running disorder correlator reaches an attractive FP of the flow equation (10) one might naively conclude from (9) and (12) that the system exhibits a usual critical scaling behavior. However, the more accurate analysis presented below for $d<d_{\mathrm{lc}}=4$ and $d>d_{\mathrm{lc}}$ shows that this is not the case.

\section{LOCALIZATION AND ACTIVATED DYNAMICS}

We start the analysis of Eqs. (8)-(12) for $d<d_{\mathrm{lc}}=4$ by studying the flow of the disorder correlator for $\Gamma_{\ell}=0$, i.e. neglecting thermal and quantum fluctuations. For concreteness we take a smooth $\pi$ - periodic bare correlator $\tilde{R}_{0}(\phi)=$ $\gamma \cos ^{2} \phi$ (the RA universality class). The flow equations for the first derivatives of $\tilde{R}_{\ell}(\phi)$ at $\phi=0$, which follow from (10), imply that the renormalized $\tilde{R}_{\ell}^{(4)}(0)$ diverges at the finite scale

$$
\ell_{c} \approx \frac{1}{\varepsilon} \ln \{1+3 \varepsilon /[8 \gamma(N+7)]\} .
$$

Beyond this scale the running disorder correlator becomes non-analytic at $\phi=0$ : the second derivative develops a cusp, $\tilde{R}_{\ell}^{\prime \prime \prime}\left(0^{+}\right) \neq 0$ for $\ell>\ell_{c}$. Then the renormalized disorder correlator $\tilde{R}_{\ell}(\phi)$ rapidly approaches a non analytic FP solution $\tilde{R}^{*}(\phi)$ with $\tilde{R}^{* \prime \prime \prime}\left(0^{+}\right) \neq 0$ and finite $\tilde{R}^{*(4)}\left(0^{+}\right)$. The stable non-analytic FP solution exists for $2 \leq N \leq N_{c}$ with $N_{c}=2.835$ for RF and $N_{c}=9.441$ for RA. These values are close to their classical limits. ${ }^{34-36}$ For instance, the $O(2)$ model has the FPs with $\tilde{R}^{* \prime \prime}(0)=-\phi_{0}^{2} \varepsilon / 36$, where $\phi_{0}=\pi$ and $\phi_{0}=2 \pi$ for the RA and RF models, respectively. The $O(3)$ and $O(4)$ RA models have the FPs with $\tilde{R}^{* \prime \prime}(0) \approx-0.309 \varepsilon$ and $\tilde{R}^{* \prime \prime}(0) \approx-0.358 \varepsilon$. The numerical $\Gamma=0$ RA FP solution for $N=3$ is shown in Fig. 1.

The numerical analysis of the full FRG flow (9)-(12) shows that the running disorder correlator $\tilde{R}_{\ell}$ can be replaced for $\ell>\ell_{c}$ by the FP point solution of the flow equation (10) at fixed $\Gamma_{\ell}$. For a finite but small $\Gamma_{\ell}$ this FP solution uniformly approaches the zero- $\Gamma$ FP solution everywhere except for the extreme points (see Fig. 1). The physically most relevant region is the boundary layer around $\phi=0$ which has the width of order $\Gamma_{\ell}$. Within the boundary layer the cusp of the zero- $\Gamma$ FP solution $\tilde{R}^{* \prime \prime}(\phi)$ is rounded by thermal and quantum fluctuations. Indeed, since $\Gamma_{\ell}$ flows towards zero the second derivative $\tilde{R}_{\ell}^{\prime \prime}(0)$ approaches $\tilde{R}^{* \prime \prime}(0) \neq 0$ while $\tilde{R}_{\ell}^{(4)}(0)$ diverges, and thus, remains different from $\tilde{R}^{*(4)}\left(0^{+}\right)$ for arbitrary small but finite $\Gamma_{\ell}$. This results in activated dynamic scaling similar to that found in the random transverse field Ising model ${ }^{37,38}$ and may lead to different behavior of averaged and typical correlations and multifractality. ${ }^{39}$ In particular the averaged connected and disconnected correlations scale differently:

$$
G_{\text {con }}(x) \sim 1 / x^{d-2+\eta}, \quad G_{\text {dis }}(x) \sim 1 / x^{d-4+\bar{\eta}}
$$

with the exponents

$$
\begin{aligned}
& \eta=-\tilde{R}^{* \prime \prime}(0), \\
& \bar{\eta}=\varepsilon-(N-1) \tilde{R}^{* \prime \prime}(0),
\end{aligned}
$$

which can be extracted from the rescaling factor (8) at the FP (for details see Ref. 36). The algebraic decay of correlators implies that the spectrum of excitations remains gapless in the whole quantum QLRO phase. This is in contrast to the pure model in the disordered phase with a gap in the energy spectrum that vanishes only at the transition to the ordered state: the quantum transition occurs when the bare effective coupling constant $g_{0}=c_{0} \tilde{\hbar}_{0}$ crosses a nontrivial FP $g^{*}=2(d-1) /(N-2)$ at zero temperature while the thermal transition takes place along the separatrix controlled by a thermal FP $g^{*}=0$ and $\tilde{T}^{*}=(d-2) /(N-2) .{ }^{28}$

To find the flow of the disorder correlator in the boundary layer we expand the flow equation (10) in small $\phi$ for fixed $\Gamma_{\ell}$. To lowest order in $\Gamma_{\ell}$ this gives $\tilde{R}_{\ell}^{\prime \prime}(0) \approx \tilde{R}^{* \prime \prime}(0)$ and

$$
\tilde{R}_{\ell}^{(4)}(0) \approx 6 \Omega /\left[\Gamma_{\ell}(N+1)\right]
$$


with the universal constant

$$
\Omega=\frac{1}{2} \tilde{R}^{* \prime \prime}(0)\left[\tilde{R}^{* \prime \prime}(0)(N-2)-\varepsilon\right] .
$$

The flow for $\ell<\ell_{c}$ is analytic and leads to renormalization of the bare parameters $\tilde{T}, \tilde{\hbar}$ and $c$ by factors of order 1 . Neglecting the latter we obtain from (9) that $\tilde{T}_{\ell}=\tilde{T}_{0} e^{-\theta\left(\ell-\ell_{c}\right)}$ and $\tilde{\hbar}_{\ell}=\tilde{\hbar}_{0} e^{-\theta_{\hbar}\left(\ell-\ell_{c}\right)}$. The exponents $\theta$ and $\theta_{\hbar}$ are given by

$$
\theta=\theta_{\hbar}-1=d-2+(N-2) \tilde{R}^{* \prime \prime}(0),
$$

to one loop order. Note that the exponent $\theta_{\hbar}$ coincides with the exponent $\theta$ in the corresponding classical system in $d+1$ dimensions with columnar disorder. We conjecture that the relation $\theta_{\hbar}=1+\theta$ holds to all orders. Substituting the disorder correlator derivatives into the boundary layer to the spin-wave velocity flow (12) and omitting the subdominant terms we find

$$
\partial_{\ell} \ln c_{\ell}=-\frac{\Omega}{\Gamma_{l}}
$$

In the classical limit $\tilde{\hbar} \rightarrow 0, \tilde{T} \rightarrow \infty$ the rounding of the cusp in the boundary layer is governed by thermal fluctuations, $\Gamma_{\ell} \approx \tilde{T}_{\ell}$. Neglecting renormalization of the spin-wave velocity $c$ below the scale $\ell_{c}$ we arrive at

$$
c_{\ell}=c_{0} e^{-\frac{\Omega}{T_{0} \theta}\left[e^{\theta\left(\ell-\ell_{c}\right)}-1\right]} .
$$

Thus, in the classical regime the low frequency spin-waves propagate via thermal activation over energy barriers that grow with the length scale $L=\Lambda_{0}^{-1} e^{\ell}$ as $L^{\theta}$. We believe that this result is also applicable to the classical $O(N)$ models with Langevin dynamics where $c$ has to be replaced by the kinetic coefficient. ${ }^{40}$ While early numerical works ${ }^{41}$ confirmed a power-law decay of correlations in the classical $O(N)$ models, recent numerical simulations ${ }^{33}$ suggested that the presence of topological defects can lead to an exponential decay of correlations on scales larger than the average distance between the defects. Thus, there is a possibility for a scenario when the dynamics is described by (21) while the algebraic decay of correlations is screened by the topological defects whose relaxation time is very large. In the opposite limit of $T \rightarrow 0$, the spin-wave velocity vanishes at a finite length scale $L_{\mathrm{loc}}=\Lambda_{0}^{-1} e^{\ell_{\mathrm{loc}}}$ with

$$
\ell_{\mathrm{loc}}-\ell_{c}=\frac{1}{\theta_{\hbar}} \ln \left[1+\frac{c_{0} \tilde{\hbar}_{0} \theta_{\hbar}}{2 \Omega}\right] .
$$

This means that the magnon excitations cannot propagate on distances larger than this scale which can be interpreted as the zero temperature spin-wave localization length. The spin and energy transport is strongly suppressed beyond this length scale leading to failure of quantum thermalization. The renormalized spin-wave velocity computed from numerical integration of the flow equation (20) for different temperatures is shown in Fig. 2. For finite but small temperature $T$ one can define an effective localization length

$$
L_{T} \approx L_{\mathrm{loc}}\left[1+\frac{\tilde{T}_{0} \theta}{\Omega} \ln \left[c_{0} \Lambda_{0} \tau_{\mathrm{exp}}\right]\right]^{1 / \theta},
$$

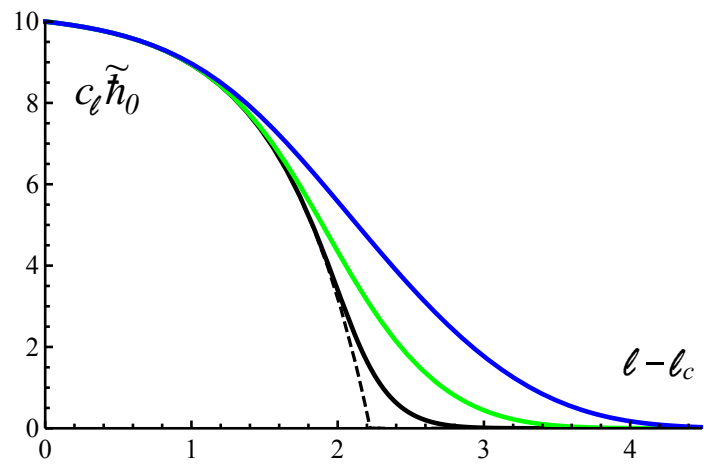

FIG. 2. (Color online) The renormalized spin-wave velocity in the 3D $O(3)$ RA model as a function of $\ell-\ell_{c}$ for different $T$ and the initial condition for the bare coupling constant $g_{0}=c_{0} \tilde{\hbar}_{0}=10$. The dashed line corresponds to $\tilde{T}_{0}=0$; the solid black, green, and blue lines to $\tilde{T}_{0}=0.2 ; 0.5 ; 1$.

beyond which the activated dynamics can be neglected on the time scale of experiment $\tau_{\text {exp }}$.

In deriving (11) we assumed that the $\Gamma_{\ell}$ is determined exclusively by the low-frequency part of the spectrum. We now show that taking into account the renormalization of the high-frequency part of the excitation spectrum leads to an extremely small but finite spin-wave velocity in the low frequency limit even at zero temperature. To see that we generalize the bare part of the effective action (4) at $T=0$ to

$$
\mathcal{S}_{n}^{(0)}=\frac{\rho_{0}}{2} \sum_{a=1}^{n} \int_{-\infty}^{\infty} \frac{d \omega}{2 \pi} \int \frac{d^{d} q}{(2 \pi)^{d}}\left[D(\omega)+q^{2}\right]\left|\mathbf{n}_{a}(\omega, q)\right|^{2} .
$$

Such generalization does not modify the flow equations for the effective Planck constant (9) and disorder correlator (10). The zero-temperature boundary layer width is, however, now given by

$$
\Gamma_{\ell}=\frac{\tilde{\hbar}_{\ell}}{\Lambda_{\ell}} \int_{-\infty}^{\infty} \frac{d \omega}{2 \pi} \frac{1}{1+\tilde{D}_{\ell}(\omega)}
$$

where we have defined $\tilde{D}_{\ell}(\omega)=\Lambda_{\ell}^{-2} D_{\ell}(\omega)$. The flow of the spectrum $\tilde{D}(\omega)$ starting from an arbitrary phononlike spectrum $\tilde{D}_{0}(\omega)$ to one loop order reads (see Appendix B)

$$
\partial_{\ell} \tilde{D}_{\ell}(\omega)=2 \tilde{D}_{\ell}(\omega)+\frac{2 \Omega}{\Gamma_{\ell}} \frac{\tilde{D}_{\ell}(\omega)}{1+\tilde{D}_{\ell}(\omega)} .
$$

Here we have retained only the terms that are relevant in the limit $\Gamma_{\ell} \rightarrow 0$. The renormalized spectrum and the boundary layer width are solutions of the self-consistent equations (25) and (26). Renormalization of the high frequency part of the spectrum (26) contributes to the boundary layer width (25) and leads to an exponentially small spin-wave velocity on scales $L>L_{\text {loc }}$. To see this we solve the spectrum flow equation (26) in the high frequency region $\tilde{D}(\omega) \gg 1$ :

$$
\tilde{D}_{\ell}(\omega) \approx e^{2 \ell} \tilde{D}_{0}(\omega)+2 \Omega \int_{\ell_{c}}^{l} \frac{d \ell^{\prime}}{\Gamma_{\ell^{\prime}}} e^{2\left(\ell-\ell^{\prime}\right)} .
$$


Plugging this in (25) and taking the bare spectrum as $\tilde{D}_{0}(\omega)=$ $\omega^{2} /\left(c_{0}^{2} \Lambda_{0}^{2}\right)$ we integrate out the high frequencies and obtain a Volterra-type integral equation for $\Gamma_{\ell}$. For large $\ell$ it can be transformed into a differential equation:

$$
\frac{d}{d \ell}\left[\frac{c_{0} \tilde{\hbar}_{\ell} e^{-\left(\ell-\ell_{c}\right)}}{2 \Gamma_{\ell}}\right]^{2}=\frac{2 \Omega}{\Gamma_{\ell}} e^{-2\left(\ell-\ell_{c}\right)},
$$

whose solution is $\Gamma_{\ell}=c_{0}^{2} \tilde{\hbar}_{\ell}^{2} \theta /(4 \Omega)$. Using the flow equation (20) we find the spin-wave velocity contribution due to renormalization of the high frequency part of the spectrum

$$
c(L) \sim \exp \left[-\frac{1+\theta}{2 \theta}\left(\frac{L}{L_{\mathrm{loc}}}\right)^{2(\theta+1)}\right],
$$

which we have expressed in terms of the localization length (22) and using the relation $\theta_{\hbar}=1+\theta$. Equation (29) shows that the effect of the residual quantum tunneling is much weaker than the effect of the thermal activation (21).

\section{ORDER-DISORDER TRANSITION}

Above the lower critical dimension $d_{\mathrm{lc}}=4$ the quantum model (2) undergoes an order-disorder transition similar to that of the classical model. ${ }^{42}$ For $\varepsilon<0$ and $N>N_{c}$ the FRG equation (10) has a FP solution which is unstable in a single direction, and thus, describes the transition. For instance, the $O(3)$ and $O(4) \mathrm{RF}$ models have the FPs with $\tilde{R}^{* \prime \prime}(0)=-5.54|\varepsilon|$ and $\tilde{R}^{* \prime \prime}(0)=-0.787|\varepsilon|$, respectively. For $N>18$ the non analyticity of the RF FP becomes weaker than a linear cusp in $\tilde{R}^{\prime \prime}(\phi)$ and its value sticks to $\tilde{R}^{* \prime \prime}(0) \approx-|\varepsilon| /(N-2)$. The large $N$ behavior of the RA FP is given by $\tilde{R}^{* \prime \prime}(0) \approx-|\varepsilon|(3 N+40) / 2(N-2)^{2}$.

The critical temperature $T_{c}(\Delta)$ is a function of the bare disorder strength (e.g. $\Delta=r^{(1)}$ for RF and $\Delta=r^{(2)}$ for RA) and vanishes at the quantum critical point $T_{c}\left(\Delta^{*}\right)=0$. The only positive eigenvalue $\lambda=|\varepsilon|$ does not depend on $N$ to one loop order and gives the critical exponent $\nu=1 / \lambda$, that describes the divergence of the correlation length in the classical regime $\xi \sim\left|T-T_{c}\right|^{-\nu}$ and at the quantum critical point $\xi \sim\left|\Delta-\Delta^{*}\right|^{-\nu}$. The hyperscaling relation between $\nu$ and the heat capacity exponent $\alpha$ is modified by the exponent $\theta$ as $\nu(d-\theta)=2-\alpha$. The averaged connected and disconnected correlation functions exhibit the power-law behavior (14) at the transition with the exponents $\eta$ and $\bar{\eta}$ related by $\bar{\eta}=2+\eta-\theta$. The critical dynamics can be studied along the same lines as for the dynamics in the QLRO phase. It turns out to be activated as well, with the typical relaxation time

$$
\tau \sim e^{C \xi^{\theta} / T}
$$

in the classical regime with $C=\Omega \rho_{0} \Lambda_{0}^{\theta-d+2} / K_{d} \theta$ and the typical relaxation time

$$
\tau \sim e^{C^{\prime} \xi^{\Psi} / \hbar^{2}}
$$

with $C^{\prime}=2 C^{2} \theta /(1+\theta)$ and

$$
\Psi=2 \theta_{\hbar}=2(\theta+1)
$$

at the quantum critical point. We expect the scaling relation (32) to hold also in the Ising case. ${ }^{22,43}$ Note that the capability of the $\varepsilon$ expansion (or other perturbative approaches such as a $2+\varepsilon$ expansion) to provide evidence for the activated classical or quantum dynamics which is expected in the random field spin systems from numerics and phenomenology has been much debated in the literature. ${ }^{22}$ Our results prove the power of the FRG method, in particular its ability to capture the activated dynamics.

\section{CONCLUSION}

We have studied the dynamics of disordered interacting quantum rotors. We found that the system is controlled by a quasiclassical zero-temperature (i.e., infinite randomness) FP with an infinite dynamic critical exponent. Below the lower critical dimension $d_{\mathrm{lc}}=4$ the system has a quantum QLRO phase with a power-law decay of correlations. At zero temperature the spin-wave excitations are localized on the length scale $L_{\mathrm{loc}}$ that prevents quantum thermalization. For $T>0$ the spin-waves propagate via thermal activation over the energy barriers which diverge in the thermodynamic dynamic limit so that the system never thermally equilibrates. These results, obtained for the $3 \mathrm{D} O(2) \mathrm{RF}$ and $O(3) \mathrm{RA}$ models, can be relevant for the quantum dynamics of the Bose glass and disordered quantum antiferromagnets.

Above the lower critical dimension the system of quantum rotors undergoes an order-disorder phase transition with activated dynamics which is strongly suppressed in the vicinity of the quantum critical point.

\section{ACKNOWLEDGMENTS}

We would like to thank L. Cugliandolo, J. Wehr, M. Gingras, T. Roscilde, D. Carpentier, E. Orignac, P. Le Doussal, and K. J. Wiese for stimulating discussions. AAF acknowledges support by ANR grants 13-JS04-0005-01 (ArtiQ) and 2010-BLANC-041902 (IsoTop).

\section{Appendix A: Derivation of the flow equations}

In order to derive the flow equations we introduce the IR cutoff by imposing a homogeneous external field $\mathbf{h}$ which is linearly coupled to $\mathbf{n}$. In the limit of small temperature and weak disorder the system is fluctuating around the completely ordered state in which all replicas of all spins align along the direction of $\mathbf{h}$. We split the order parameter $\mathbf{n}_{a}=\left(\sigma_{a}, \boldsymbol{\pi}_{a}\right)$ into the $(N-1)$-component vector $\boldsymbol{\pi}_{a}$ which is perpendicular to $\mathbf{h}$ and the component $\sigma_{a}=\sqrt{1-\pi_{a}^{2}}$ parallel to it. Then the partition function can be rewritten as

$$
Z=\int \prod_{a=1}^{n} \mathcal{D} \pi_{a} \prod_{\tau, x} \frac{1}{\sqrt{1-\pi_{a}^{2}(\tau, x)}} e^{-\mathcal{S}_{n}[\boldsymbol{\pi}] / \hbar}
$$


with the replicated action

$$
\begin{aligned}
\mathcal{S}_{n}[\boldsymbol{\pi}] & =\frac{\rho_{0}}{2} \int_{x, \tau} \sum_{a=1}^{n}\left\{\frac{1}{c_{0}^{2}}\left[\left(\partial_{\tau} \boldsymbol{\pi}_{a}\right)^{2}+\frac{\left(\boldsymbol{\pi}_{a} \cdot \partial_{\tau} \boldsymbol{\pi}_{a}\right)^{2}}{\left(1-\boldsymbol{\pi}_{a}^{2}\right)}\right]\right. \\
+ & \left.\left(\nabla \boldsymbol{\pi}_{a}\right)^{2}+\frac{\left(\boldsymbol{\pi}_{a} \cdot \nabla \boldsymbol{\pi}_{a}\right)^{2}}{\left(1-\boldsymbol{\pi}_{a}^{2}\right)}-h \sqrt{1-\boldsymbol{\pi}_{a}^{2}}\right\} \\
& -\frac{1}{2 \hbar} \sum_{a, b=1}^{n} \int_{x, \tau, \tau^{\prime}} R\left(\boldsymbol{\pi}_{a}(\tau, x) \cdot \boldsymbol{\pi}_{b}\left(\tau^{\prime}, x\right)\right. \\
& \left.+\sigma_{a}(\tau, x) \sigma_{b}\left(\tau^{\prime}, x\right)\right) .
\end{aligned}
$$

We use the momentum shell method developed in Refs. 28 and 44 and consider the loop expansion in small $\hbar$ and $R$. To that end we express $\pi_{a}$ as

$$
\boldsymbol{\pi}_{a}(\tau, x)=\sum_{m=-\infty}^{\infty} \int \frac{d^{d} q}{(2 \pi)^{d}} \boldsymbol{\pi}_{a}\left(\omega_{m}, q\right) e^{i \omega_{m} \tau+i q \cdot x}
$$

where we have introduced the Matsubara frequencies $\omega_{m}=$ $2 \pi T m / \hbar$ with $m \in \mathbb{Z}$. We now decompose the fields $\boldsymbol{\pi}_{a}$ into slowly and rapidly varying parts as follows

$$
\boldsymbol{\pi}_{a}\left(\omega_{m}, q\right)= \begin{cases}\boldsymbol{\pi}_{a}^{<}\left(\omega_{m}, q\right), & 0<q<\Lambda_{\ell} \\ \boldsymbol{\pi}_{a}^{>}\left(\omega_{m}, q\right), & \Lambda_{\ell}<q<\Lambda_{0} .\end{cases}
$$

Integrating out $\boldsymbol{\pi}_{a}^{>}$and rescaling momenta by $e^{\ell}$ and the fields $\pi_{a}^{<}$by $\zeta$ we obtain the effective action of the same form (A2) which involves only $\boldsymbol{\pi}_{a}^{<}$and the new parameters $T^{\prime}, c^{\prime}, \hbar^{\prime}, h^{\prime}$ and $[R(z)]^{\prime}$. It is convenient to introduce $\hat{R}(\phi)=R(z)$ with $z=\cos \phi$. The bare disorder correlator $R(z)$ is an analytic function of $z$ for $-1 \leq z \leq 1$. However, the renormalized disorder correlator becomes nonanalytic around $z=1$ and as can be checked a posteriori by solving the flow equation it has the following expansion: ${ }^{34}$

$$
\begin{aligned}
R(z)= & R(1)+R^{\prime}(1)(z-1)+\frac{a_{1}}{3}[2(1-z)]^{3 / 2} \\
& +\frac{a_{2}}{2}(z-1)^{2}+\cdots,
\end{aligned}
$$

which corresponds to

$\hat{R}(\phi)=\hat{R}(0)+\frac{\hat{R}^{\prime \prime}(0)}{2} \phi^{2}+\frac{\hat{R}^{\prime \prime \prime}\left(0^{+}\right)}{3 !} \phi^{3}+\frac{\hat{R}^{(4)}(0)}{4 !} \phi^{3}+\cdots$

with

$$
\begin{aligned}
& \hat{R}^{\prime \prime}(0)=-R^{\prime}(1), \\
& \hat{R}^{\prime \prime \prime}(0)=2 a_{1}, \\
& \hat{R}^{(4)}(0)=R^{\prime}(1)+3 a_{2} .
\end{aligned}
$$

\section{Renormalization of the single-replica terms}

From the one-loop correction to the term $\left(\nabla \pi_{a}\right)^{2}$ we find

$$
\frac{1}{\hbar^{\prime}}=\frac{\zeta^{2}}{\hbar}\left[1+\hbar J_{1}+R^{\prime}(1) J_{2}\right]
$$

where the one-loop integrals are given by

$$
\begin{aligned}
J_{1}= & \frac{K_{d} T}{\rho \hbar} \sum_{m=-\infty}^{\infty} \int_{\Lambda e^{-\ell}}^{\Lambda} \frac{q^{d-1} d q}{q^{2}+c^{-2} \omega_{m}^{2}} \\
& =\frac{K_{d}}{2 \rho} c \Lambda^{d-1}\left(1-e^{-\ell}\right) \operatorname{coth}\left[\frac{c \hbar \Lambda}{2 T}\right]
\end{aligned}
$$

and

$$
J_{2}=\frac{K_{d}}{\rho^{2}} \int_{\Lambda e^{-\ell}}^{\Lambda} \frac{q^{d-1} d q}{q^{4}}=\frac{K_{d}}{\rho^{2}} \frac{\Lambda^{d-4}}{d-4}\left[1-e^{-\ell(d-4)}\right] .
$$

The correction to the external field reads

$$
\frac{h^{\prime}}{\hbar^{\prime}}=\zeta^{2}\left(\frac{h}{\hbar}\right)\left[1+\frac{1}{2}(N-1)\left[\hbar J_{1}+R^{\prime}(1) J_{2}\right]\right] .
$$

The spin rescaling factor $\zeta$ can be found by noting that the combination $h / \hbar$ renormalizes trivially as ${ }^{44} h^{\prime} / \hbar^{\prime}=\zeta(h / \hbar)$. This gives

$$
\zeta=1-\frac{1}{2}(N-1)\left[\hbar J_{1}+R^{\prime}(1) J_{2}\right]
$$

and

$$
\hbar^{\prime}=\hbar\left[1+(N-2)\left(\hbar J_{1}+R^{\prime}(1) J_{2}\right)\right] .
$$

The renormalization of the spin wave velocity can be found from the correction to the $\left(\partial_{\tau} \boldsymbol{\pi}_{a}\right)^{2}$ term:

$$
\begin{aligned}
\frac{\left(\omega_{m}^{\prime}\right)^{2}}{c^{\prime 2} \hbar^{\prime}}= & \zeta^{2}\left(\frac{\omega^{2}}{c^{2} \hbar}\right)\left[1+\hbar J_{1}+2 R^{\prime}(1) J_{2}\right. \\
& \left.+(N+1) a_{2} J_{2}\right],
\end{aligned}
$$

which can be rewritten as

$$
\begin{aligned}
\frac{c^{\prime} \hbar^{\prime}}{T^{\prime}}= & \frac{c \hbar}{T}\left[1-\frac{1}{6}(N+1) \hat{R}^{(4)}(0) J_{2}\right. \\
& \left.-\frac{1}{6}(N-2) \hat{R}^{\prime \prime}(0) J_{2}\right] .
\end{aligned}
$$

Using the definitions (5)-(7) we derive from Eqs. (A13)-(A17) the flow equations (8) and (9).

\section{Correction to disorder}

The disorder term contains two replicas: To find its renormalization it is convenient to expand around some background state $\mathbf{n}_{a}^{0}(\tau, x)$ which depends explicitly on replica index $a$ and slowly changes in space. ${ }^{35}$ For a particular pair of replicas $a$ and $b$ we reparametrize $\mathbf{n}_{a}(\tau, x)$ and $\mathbf{n}_{b}(\tau, x)$ as $\mathbf{n}_{a}(\tau, x)=$ $\left(\sigma_{a}, \eta_{a}, \boldsymbol{\rho}_{a}\right)$ and $\mathbf{n}_{b}(\tau, x)=\left(\sigma_{b}, \eta_{b}, \boldsymbol{\rho}_{b}\right)$ where the $\sigma$ and $\eta$ components lie in the plane spanned by vectors $\mathbf{n}_{a}^{0}$ and $\mathbf{n}_{b}^{0}$ in a such way that $\sigma_{a}=\sqrt{1-\eta_{a}^{2}-\boldsymbol{\rho}_{a}^{2}}$ is parallel to $\mathbf{n}_{a}^{0}$ and $\sigma_{b}=\sqrt{1-\eta_{b}^{2}-\boldsymbol{\rho}_{b}^{2}}$ to $\mathbf{n}_{b}^{0}$. The components $\boldsymbol{\rho}$ are orthogonal to the plane. Defining the angle between $\mathbf{n}_{a}^{0}$ and $\mathbf{n}_{b}^{0}$ as $\phi_{a b}$ we obtain $\mathbf{n}_{a} \cdot \mathbf{n}_{b}=\boldsymbol{\rho}_{a} \cdot \boldsymbol{\rho}_{b}+\cos \phi_{a b}\left(\sigma_{a} \sigma_{b}+\eta_{a} \eta_{b}\right)+$ $\sin \phi_{a b}\left(\sigma_{a} \eta_{b}-\sigma_{b} \eta_{a}\right)$. Expanding in small $\eta$ and $\rho$ we get

$$
\begin{aligned}
& \mathbf{n}_{a} \cdot \mathbf{n}_{b}=\boldsymbol{\rho}_{a} \cdot \boldsymbol{\rho}_{b}+\cos \phi_{a b}\left[1-\frac{1}{2}\left(\eta_{a}^{2}+\boldsymbol{\rho}_{a}^{2}\right.\right. \\
& \left.\left.+\eta_{b}^{2}+\boldsymbol{\rho}_{b}^{2}\right)+\eta_{a} \eta_{b}\right]+\sin \phi_{a b} \\
& \times\left[\eta_{b}-\eta_{a}-\frac{1}{2} \eta_{b}\left(\eta_{a}^{2}+\boldsymbol{\rho}_{a}^{2}\right)+\frac{1}{2} \eta_{a}\left(\eta_{b}^{2}+\boldsymbol{\rho}_{b}^{2}\right)\right] .
\end{aligned}
$$


Substituting Eq. (A18) into $R$ and expanding again in small $\eta$ and $\rho$ to second order we obtain

$$
\begin{aligned}
R\left(\mathbf{n}_{a} \cdot \mathbf{n}_{b}\right)= & R\left(\cos \phi_{a b}\right)+R^{\prime}\left(\cos \phi_{a b}\right)\left\{\boldsymbol{\rho}_{a} \cdot \boldsymbol{\rho}_{b}\right. \\
& \left.-\frac{1}{2} \cos \phi_{a b}\left[\boldsymbol{\rho}_{a}^{2}+\boldsymbol{\rho}_{b}^{2}+\left(\eta_{a}-\eta_{b}\right)^{2}\right]\right\} \\
& +\frac{1}{2} R^{\prime \prime}\left(\cos \phi_{a b}\right)\left(\eta_{a}-\eta_{b}\right)^{2} \sin ^{2} \phi_{a b} .
\end{aligned}
$$

Using that $R(\cos \phi)=\hat{R}(\phi), R^{\prime}(\cos \phi)=-\hat{R}^{\prime}(\phi) / \sin \phi$ and $R^{\prime \prime}(\cos \phi)=\left[\hat{R}^{\prime \prime}(\phi)-\hat{R}^{\prime}(\phi) \cos \phi / \sin \phi\right] / \sin ^{2} \phi$ we can rewrite Eq. (A19) as

$$
\begin{aligned}
R\left(\mathbf{n}_{a} \cdot \mathbf{n}_{b}\right)= & \hat{R}\left(\phi_{a b}\right)+\hat{R}^{\prime}\left(\phi_{a b}\right)\left\{\frac{\boldsymbol{\rho}_{a}^{2}+\boldsymbol{\rho}_{b}^{2}}{2 \tan \phi_{a b}}-\frac{\boldsymbol{\rho}_{a} \cdot \boldsymbol{\rho}_{b}}{\sin \phi_{a b}}\right\} \\
& +\frac{1}{2} \hat{R}^{\prime \prime}\left(\phi_{a b}\right)\left(\eta_{a}-\eta_{b}\right)^{2} .
\end{aligned}
$$

To compute the one-loop correction to the disorder correlator we expand $\exp [-\mathcal{S} / \hbar]$ to second order in $R\left(\mathbf{n}_{a} \cdot \mathbf{n}_{b}\right)$ and perform Gaussian integration over $\eta$ and $\boldsymbol{\rho}$ assuming that they contain only the fast parts of $\boldsymbol{\pi}_{a}$. To extract the one-loop correction it is enough to keep the terms quartic in $\eta$ and $\rho$, which gives

$$
\begin{aligned}
& \delta^{(1)}\left[\frac{\hat{R}\left(\phi_{a b}\right)}{2 \hbar^{2}}\right]=\frac{1}{8 \hbar^{4}} \sum_{a b c d}\left\{\frac{1}{4} \hat{R}^{\prime \prime}\left(\phi_{a b}\right) \hat{R}^{\prime \prime}\left(\phi_{c d}\right)\right. \\
& \times\left\langle\left(\eta_{a}-\eta_{b}\right)^{2}\left(\eta_{c}-\eta_{d}\right)^{2}\right\rangle+\hat{R}^{\prime}\left(\phi_{a b}\right) \hat{R}^{\prime}\left(\phi_{c d}\right) \\
& \left.\left\langle\left(\frac{\boldsymbol{\rho}_{a}^{2}+\boldsymbol{\rho}_{b}^{2}}{2 \tan \phi_{a b}}-\frac{\boldsymbol{\rho}_{a} \cdot \boldsymbol{\rho}_{b}}{\sin \phi_{a b}}\right)\left(\frac{\boldsymbol{\rho}_{c}^{2}+\boldsymbol{\rho}_{d}^{2}}{2 \tan \phi_{c d}}-\frac{\boldsymbol{\rho}_{c} \cdot \boldsymbol{\rho}_{d}}{\sin \phi_{c d}}\right)\right\rangle\right\} .
\end{aligned}
$$

Since the integration over $\eta$ and $\rho$ is Gaussian we can use the Wick theorem with the following contractions:

$$
\begin{aligned}
\left\langle\rho_{a}^{i}(q, \omega) \rho_{b}^{j}(-q,-\omega)\right\rangle & =\frac{\hbar \delta^{i j} \delta_{a b}}{q^{2}+c_{0}^{-2} \omega^{2}+h}, \\
\left\langle\eta_{a}(q, \omega) \eta_{b}(-q,-\omega)\right\rangle & =\frac{\hbar \delta_{a b}}{q^{2}+c_{0}^{-2} \omega^{2}+h} .
\end{aligned}
$$

Using this in Eq. (A21) we obtain one loop diagrams in which there is non-zero momentum circulation while there is no frequency circulation since the disorder vertices do not transmit it. For instance we have

$$
\left\langle\left(\eta_{a}-\eta_{b}\right)^{2}\left(\eta_{c}-\eta_{d}\right)^{2}\right\rangle=8 \hbar^{2} J_{2} \delta_{a c} \delta_{b d}-16 \hbar^{2} J_{2} \delta_{a c} \delta_{a d},
$$

which can be depicted using the following diagrams

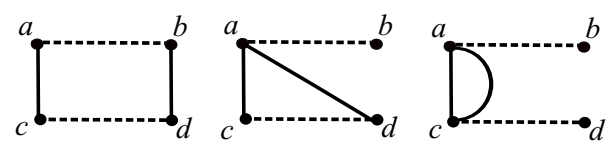

The last diagram is proportional to $\delta_{a c} \delta_{a c}$. After summation over replica indices it gives the number of replicas, and thus, vanishes in the limit of zero replicas $n \rightarrow 0$. Similarly we obtain

$$
\left\langle\left(\boldsymbol{\rho}_{a} \cdot \boldsymbol{\rho}_{b}\right)\left(\boldsymbol{\rho}_{c} \cdot \boldsymbol{\rho}_{d}\right)\right\rangle=2(N-2) \hbar^{2} J_{2} \delta_{a c} \delta_{b d},
$$

and

$$
\frac{\hat{R}^{\prime}\left(\phi_{c d}\right)}{\sin \phi_{c d}}\left\langle\left(\boldsymbol{\rho}_{a}^{2}\right)\left(\boldsymbol{\rho}_{c} \cdot \boldsymbol{\rho}_{d}\right)\right\rangle=2(N-2) \hbar^{2} J_{2} \delta_{a c} \delta_{b d} \frac{\hat{R}^{\prime}\left(\phi_{a a}\right)}{\sin \phi_{a a}},
$$

where we can replace $\hat{R}^{\prime}\left(\phi_{a a}\right) / \sin \phi_{a a}$ by $\hat{R}^{\prime \prime}(0)$ using that $\phi_{a a}=0$. Denoting $\phi:=\phi_{a b}$ we arrive at

$$
\begin{aligned}
& \delta^{(1)}\left[\frac{\hat{R}(\phi)}{2 \hbar^{2}}\right]=\frac{1}{2 \hbar^{2}}\left\{\frac{1}{2}\left[\hat{R}^{\prime \prime}(\phi)\right]^{2}-\hat{R}^{\prime \prime}(\phi) \hat{R}^{\prime \prime}(0)\right. \\
& \left.+(N-2)\left(\frac{1}{2} \frac{\hat{R}^{\prime}(\phi)^{2}}{\sin ^{2} \phi}-\frac{\hat{R}^{\prime}(\phi) \hat{R}^{\prime \prime}(0)}{\tan \phi}\right)\right\} J_{2} .
\end{aligned}
$$

The correction to the disorder correlator due to finite $\hbar$ is given by

$$
\begin{aligned}
\delta^{(2)}\left[\frac{\hat{R}(\phi)}{2 \hbar^{2}}\right]= & \left\langle-R^{\prime}\left(\cos \phi_{a b}\right) \cos \phi_{a b}\left[\boldsymbol{\rho}_{b}^{2}+\eta_{b}^{2}\right]\right. \\
& \left.+R^{\prime \prime}\left(\cos \phi_{a b}\right) \sin ^{2} \phi_{a b} \eta_{b}^{2}\right\rangle \\
& =\hbar J_{1}\left[R^{\prime}\left(\cos \phi_{a b}\right)\left\{-\cos \phi_{a b}[(N-2)+1]\right\}\right. \\
& \left.+R^{\prime \prime}\left(\cos \phi_{a b}\right) \sin ^{2} \phi_{a b}\right] \\
& =\hbar J_{1}\left[(N-2) \frac{\hat{R}^{\prime}(\phi)}{\tan \phi}+\hat{R}^{\prime \prime}(\phi)\right] .
\end{aligned}
$$

Using

$$
\frac{[\hat{R}(\phi)]^{\prime}}{2 \hbar^{\prime 2}}=\frac{\hat{R}(\phi)}{2 \hbar^{2}}+\delta^{(1)}\left[\frac{\hat{R}(\phi)}{2 \hbar^{2}}\right]+\delta^{(2)}\left[\frac{\hat{R}(\phi)}{2 \hbar^{2}}\right](
$$

and Eq. (A15) we derive

$$
\begin{aligned}
& {[\hat{R}(\phi)]^{\prime}=\hat{R}(\phi)+\hat{R}^{\prime \prime}(\phi)\left[\hbar J_{1}-\hat{R}^{\prime \prime}(0) J_{2}\right]} \\
& +\frac{1}{2}\left[\hat{R}^{\prime \prime}(\phi)\right]^{2} J_{2}+(N-2)\left(\frac{1}{2} \frac{\hat{R}^{\prime}(\phi)^{2}}{\sin ^{2} \phi} J_{2}\right. \\
& \left.+\left[\frac{\hat{R}^{\prime}(\phi)}{\tan \phi}+2 \hat{R}(\phi)\right]\left[\hbar J_{1}-\hat{R}^{\prime \prime}(0) J_{2}\right]\right),
\end{aligned}
$$

where $J_{1}$ and $J_{2}$ are given by Eqs. (A11) and (A12). The flow equation for the disorder correlator (10) follows from Eq. (A26).

\section{Appendix B: Renormalization of the entire spectrum}

Renormalization of the generalized action (24) resembles renormalization of the clean NL $\sigma \mathrm{M}$ with a damping term, ${ }^{45}$

$$
D_{0}(\omega)=\frac{\omega^{2}}{c^{2}}+f(\omega)
$$

The particular form of the damping term $f(\omega)$ depends on the mechanism of damping and it has to be introduced into the clean NL $\sigma \mathrm{M}$ by hand. ${ }^{45}$ In our model the form of the spectrum 
is renormalized by disorder. Indeed, the one-loop correction to $D(\omega)$ reads

$$
\begin{aligned}
\frac{[D(\omega)]^{\prime}}{\hbar^{\prime}}= & \frac{\zeta^{2}}{\hbar}\left\{D(\omega)+\hbar I_{2}(\omega)+R^{\prime}(1)\left[D(\omega) J_{2}+I_{1}(\omega)\right]\right. \\
& \left.+a_{2}(N+1) I_{1}(\omega)\right\},
\end{aligned}
$$

where

$$
\begin{aligned}
& I_{1}(\omega)=\frac{1}{\rho^{2}} \int_{q}\left[\frac{1}{q^{2}+h}-\frac{1}{q^{2}+D(\omega)+h}\right], \\
& I_{2}(\omega)=\frac{1}{\rho} \sum_{m=-\infty}^{\infty} \int_{q} \frac{D\left(\omega+\omega_{m}\right)-D\left(\omega_{m}\right)}{q^{2}+D\left(\omega_{m}\right)+h} .
\end{aligned}
$$

Using Eqs. (A15) and (B2) we obtain

$$
\begin{aligned}
{[D(\omega)]^{\prime}=} & D(\omega)+\hbar\left[I_{2}(\omega)-D(\omega) J_{1}\right] \\
& +\left[R^{\prime}(1)+a_{2}(N+1)\right] I_{1}(\omega) .
\end{aligned}
$$

The first term $\hbar\left[I_{2}(\omega)-D(\omega) J_{1}\right]$ which was found for the pure $\mathrm{NL} \sigma \mathrm{M}$ in Ref. 45 is irrelevant at the zero temperature quasi-classical FP. The integral in the second term gives

$$
\begin{aligned}
I_{1}(\omega)= & \frac{K_{d}}{\rho^{2}} \int_{\Lambda e^{-\ell}}^{\Lambda} \frac{D(\omega) q^{d-1} d q}{q^{2}\left[q^{2}+D(\omega)\right]} \\
& =\frac{K_{d}}{\rho^{2}} \frac{D(\omega) \Lambda^{d-2} \ell}{\Lambda^{2}+D(\omega)} .
\end{aligned}
$$

Introducing $\tilde{D}(\omega)=\Lambda_{\ell}^{-2} D(\omega)$ and using Eq. (5) we find the flow equation for the spectrum as

$$
\begin{aligned}
\partial_{\ell} \tilde{D}(\omega)= & 2 \tilde{D}(\omega)+\frac{1}{3}\left[(N+1) \tilde{R}^{(4)}(0)\right. \\
& \left.+(N-2) \tilde{R}^{\prime \prime}(0)\right] \frac{\tilde{D}(\omega)}{1+\tilde{D}(\omega)} .
\end{aligned}
$$

Retaining in Eq. (B7) only the terms which are dominant in the vicinity of a zero temperature quasi-classical FP we arrive at Eq. (26).
${ }^{1}$ F. Evers and A. D. Mirlin, Rev. Mod. Phys. 80, 1355 (2008).

2 D. M. Basko, I. L. Aleiner, and B. L. Altshuler, Annals of Physics 321, 1126 (2006).

3 I. L. Aleiner, B. L. Altshuler, and G. V. Shlyapnikov, Nature Physics 6, 900 (2010).

4 X. Yu and M. Mueller, Annals of Physics 337, 55 (2013).

5 A. Pal and D. A. Huse, Phys. Rev. B 82, 174411 (2010).

${ }^{6}$ R. Vosk and E. Altman, Phys. Rev. Lett. 110, 067204 (2013).

7 M. P. A. Fisher, P. B. Weichman, G. Grinstein, and D. S. Fisher, Phys. Rev. B 40, 546 (1989).

8 D. R. Nelson and V. M. Vinokur, Phys. Rev. Lett. 68, 2398 (1992).

9 T. Giamarchi and P. Le Doussal, Phys. Rev. B 53, 15206 (1996).

10 A. A. Fedorenko, Phys. Rev. B 77, 094203 (2008).

${ }^{11}$ R. Yu, L. Yin, N. S. Sullivan, J. S. Xia, C. Huan, A. PaduanFilho, N. F. Oliveira, Jr., S. Haas, A. Steppke, C. F. Miclea, F. Weickert, R. Movshovich, E.-D. Mun, B. L. Scott, V. S. Zapf, and T. Roscilde, Nature 489, 379 (2012).

12 E. Orignac, T. Giamarchi, and P. Le Doussal, Phys. Rev. Lett. 83, 2378 (1999).

13 T. Giamarchi, P. Le Doussal, and E. Orignac, Phys. Rev. B 64, 245119 (2001).

14 J. P. A. Zúñiga and N. Laflorencie, Phys. Rev. Lett. 111, 160403 (2013).

15 A. Gangopadhyay, V. Galitski, and M. Müller, Phys. Rev. Lett. 111, 026801 (2013).

16 D. S. Fisher, Phys. Rev. Lett. 56, 1964 (1986).

17 T. Nattermann, S. Stepanow, L.-H. Tang, and H. Leschhorn, J. Phys. II France 2, 1483 (1992).

18 P. Chauve, P. Le Doussal, and K. J. Wiese, Phys. Rev. Lett. 86, 1785 (2001).

19 A. A. Fedorenko, P. Le Doussal, and K. J. Wiese, Phys. Rev. E 74, 061109 (2006).

${ }^{20}$ D. S. Fisher, Phys. Rev. Lett. 56, 416 (1986).

21 A. Dutta and J. K. Bhattacharjee, Phys. Rev. B 58, 6378 (1998).
22 T. Senthil, Phys. Rev. B 57, 8375 (1998).

23 P. Chauve, T. Giamarchi, and P. Le Doussal, Phys. Rev. B 62, 6241 (2000).

${ }^{24}$ D. A. Gorokhov, D. S. Fisher, and G. Blatter, Phys. Rev. B 66, 214203 (2002).

25 J. Ye, S. Sachdev, and N. Read, Phys. Rev. Lett. 70, 4011 (1993).

26 A. Andreanov and M. Müller, Phys. Rev. Lett. 109, 177201 (2012).

27 M. A. Cazalilla, R. Citro, T. Giamarchi, E. Orignac, and M. Rigol, Rev. Mod. Phys. 83, 1405 (2011).

28 S. Chakravarty, B. I. Halperin, and D. R. Nelson, Phys. Rev. B 39, 2344 (1989).

29 E. Demler, W. Hanke, and S.-C. Zhang, Rev. Mod. Phys. 76, 909 (2004).

30 D. S. Fisher, Phys. Rev. B 31, 7233 (1985).

31 A. A. Fedorenko and F. Kühnel, Phys. Rev. B 75, 174206 (2007).

${ }^{32}$ D. E. Feldman, Phys. Rev. B 61, 382 (2000).

33 T. C. Proctor, D. A. Garanin, and E. M. Chudnovsky, Phys. Rev. Lett. 112, 097201 (2014).

${ }^{34}$ M. Tissier and G. Tarjus, Phys. Rev. B 74, 214419 (2006).

35 P. Le Doussal and K. J. Wiese, Phys. Rev. Lett. 96, 197202 (2006).

36 A. A. Fedorenko, Phys. Rev. E 86, 021131 (2012).

37 A. P. Young and H. Rieger, Phys. Rev. B 53, 8486 (1996).

38 C. Monthus and T. Garel, Journal of Physics A: Mathematical and Theoretical 45, 095002 (2012).

39 A. A. Fedorenko, P. Le Doussal, and K. J. Wiese, EPL 105, 16002 (2014).

40 A. A. Fedorenko and S. Trimper, Europhys. Lett. 74, 89 (2006).

${ }^{41}$ M. Itakura, Phys. Rev. B 68, 100405 (2003).

${ }^{42}$ D. E. Feldman, Phys. Rev. Lett. 88, 177202 (2002).

${ }^{43}$ F. Anfuso and A. Rosch, Eur. Phys. J. B 69, 465 (2009).

44 D. R. Nelson and R. A. Pelcovits, Phys. Rev. B 16, 2191 (1977).

45 A. Gamba, M. Grilli, and C. Castellani, Nuclear Physics B 556, 463 (1999). 